\title{
Failure patterns and related risk factors of sagittal reconstruction following pedicle subtraction osteotomy in patients with ankylosing spondylitis and thoracolumbar kyphosis
}

\author{
Shi-Zhou Zhao, MD,,2, Bang-Ping Qian, MD,1 Ji-Chen Huang, MD,, ${ }^{1,2}$ Mu Qiao, MD,1 Bin Wang, MD,1 \\ and Yong Qiu, MD'
}

1Spine Surgery, Affiliated Drum Tower Hospital, Medical School of Nanjing University, Nanjing; and ${ }^{2}$ Medical School of Nanjing University, Nanjing, China

OBJECTIVE The aim of this study was to analyze the specific patterns and risk factors of sagittal reconstruction failure in ankylosing spondylitis (AS)-related thoracolumbar kyphosis after pedicle subtraction osteotomy (PSO).

METHODS A retrospective study was performed in patients with AS and thoracolumbar kyphosis after lumbar PSO with a minimum follow-up of 2 years. Patients were classified as having successful realignment (group A), inadequate correction immediately postoperatively (group B), and sagittal decompensation during follow-up (group C) according to the immediately postoperative and latest follow-up sagittal vertical axis (SVA). Radiographic parameters and clinical outcomes were collected. Pelvic tilt (PT) was used to assess the magnitude of pelvic backward rotation. Hip structural damage and ossification of the anterior longitudinal ligament (ALL) at the proximal junction, PSO level, and distal junction were also evaluated on radiographs.

RESULTS Overall, 109 patients with a mean age of 35.3 years were included. Patients in both group $B(n=16)$ and group $C(n=13)$ were older than those in group $A(n=80)$ (mean ages 43.6 vs 32.9 years, $p<0.011$; and 39.2 vs 32.9 years, $p=0.018$; respectively). Age (OR 1.102, $p=0.011$ ), and preoperative PT (OR 1.171, $p=0.041$ ) and SVA (OR 1.041, $p=0.016$ ) were identified as independent risk factors of inadequate correction. Additionally, a higher distribution of patients with adequate ALL ossification at the PSO level was found in group B than in group A ( $37.5 \%$ vs $22.5 \%, p=$ 0.003). Age (OR 1.101, $p=0.011$ ) and preoperative SVA (OR 1.013, $p=0.020$ ) were identified as independent risk factors of sagittal decompensation. Furthermore, compared with group $\mathrm{A}$, group $\mathrm{C}$ showed a higher distribution of patients with severe hip structural damage $(15.4 \%$ vs $0, p=0.018)$ and higher incidences of rod fracture (RF) $(38.5 \%$ vs $8.8 \%, p$ $=0.011)$ and pseudarthrosis (15.4\% vs $0, p=0.018)$. Additionally, the incidence of $\operatorname{RF}(19.6 \%$ vs $6.9 \%, p=0.045)$ and changes in the proximal junctional angle $\left(0.5^{\circ}\right.$ vs $\left.2.2^{\circ}, p=0.027\right)$ and the distal junctional angle $\left(0.3^{\circ}\right.$ vs $\left.2.2^{\circ}, p=0.019\right)$ were lower during follow-up in patients with adequate ALL ossification than in those without adequate ossification.

CONCLUSIONS Sagittal reconstruction failure in patients with AS could be attributed to inadequate correction immediately after surgery (14.7\%) and sagittal decompensation during follow-up (11.9\%). Adequate ALL ossification was a risk factor of inadequate correction. However, adequate ALL ossification could decrease the development of RF and relieve the junctional kyphotic change during follow-up. Older age and greater baseline SVA were independent risk factors for both inadequate correction and sagittal decompensation.

https://thejns.org/doi/abs/10.3171/2021.7.FOCUS21146

KEYWORDS ankylosing spondylitis; thoracolumbar kyphosis; pedicle subtraction osteotomy; sagittal reconstruction failure; inadequate correction; sagittal decompensation

ABBREVIATIONS ALL = anterior longitudinal ligament; AS = ankylosing spondylitis; ASD = adult spinal deformity; BASRI-h = Bath Ankylosing Spondylitis Radiology Index of the hip; DJA = distal junctional angle; DJK = distal junctional kyphosis; HRQOL = health-related quality of life; LIV = lower instrumented vertebra; LL = lumbar lordosis; $\mathrm{ODI}=$ Oswestry Disability Index; OVA = osteotomy vertebra angle; $\mathrm{PI}=$ pelvic incidence; $\mathrm{PJA}=$ proximal junctional angle; $\mathrm{PJK}=$ proximal junctional kyphosis; $\mathrm{PSO}=$ after pedicle subtraction osteotomy; $\mathrm{PT}=$ pelvic tilt; RF = rod fracture; $\mathrm{SS}=$ sacral slope; $\mathrm{ST}$ = sagittal translation; $\mathrm{SVA}=$ sagittal vertical axis; $\mathrm{THA}=$ total hip arthroplasty; TK = thoracic kyphosis; UIV = upper instrumented vertebra; VAS = visual analog scale.

SUBMITTED March 2, 2021. ACCEPTED July 13, 2021

INCLUDE WHEN CITING DOI: 10.3171/2021.7.FOCUS21146. 
A NKYLOSING spondylitis (AS) is a chronic inflammatory disease that mainly affects the spine and hip. ${ }^{1}$ Specifically, spine changes are characterized by heterotopic ossification of the facet joints and paravertebral ligaments. ${ }^{2}$ In the advanced stage of AS, patients have difficulty maintaining a standing upright posture and horizontal gaze due to the rigid thoracolumbar kyphosis. ${ }^{2}$ Pedicle subtraction osteotomy (PSO) has been proven to be an effective sagittal correction approach for AS-related thoracolumbar kyphosis. ${ }^{2,3}$ Moreover, the postoperative health-related quality of life (HRQOL) for patients with AS is positively correlated with sagittal alignment following PSO. ${ }^{3}$

Despite the substantial deformity correction after PSO, undercorrection and mechanical complications, including junctional kyphosis and rod fracture (RF), have been reported in AS.,5 Theoretically, both inadequate correction immediately after surgery and mechanical complications during follow-up could lead to sagittal reconstruction failure. As previously reported in adult spinal deformity (ASD), the rate of sagittal reconstruction failure due to inadequate correction ranged from $11 \%$ to $33 \%, 6,7$ with greater preoperative spinal deformity having been reported as a risk factor. ${ }^{8}$ Additionally, mechanical complications of junctional kyphosis, screw loosening, and RF during follow-up have also been confirmed as causes of sagittal decompensation after ASD correction surgery. Moreover, the rate of sagittal malalignment recurrence could be up to $38 \%,{ }^{6,9,10}$ and the risk factors include age, upper instrumented vertebra (UIV) level, lower instrumented vertebra (LIV) level, fusion levels, and the magnitude of deformity correction. ${ }^{11,12}$ Recently, the role of spinal mobility in the development of mechanical complications during follow-up has been emphasized. ${ }^{13}$ Considering the restricted spinal motion due to heterotopic ossification in AS, the rates and types of mechanical complications might differ from those in ASD. Moreover, ossification of the anterior longitudinal ligament (ALL) at the PSO level would impair the lordosing effect of the neighboring disc opening, potentially resulting in inadequate correction. ${ }^{14}$ Hence, the rates and patterns of sagittal reconstruction failure in AS might be different from that in ASD due to the heterotopic ossification.

To the best of our knowledge, few articles have focused on sagittal reconstruction failure in AS-related thoracolumbar kyphosis. Therefore, the purpose of this study was to analyze the specific patterns and risk factors of sagittal reconstruction failure in AS-related thoracolumbar kyphosis after PSO. The role of ossification of the ALL was also investigated.

\section{Methods \\ Patients}

A retrospective review of patients with AS and thoracolumbar kyphosis following one-level lumbar PSO at our center from January 2011 to April 2018 was performed. This study was approved by the IRB, and informed consent was obtained from all patients. Inclusion criteria were 1) patients with $\mathrm{AS}>18$ years of age; 2) a minimum of 2 years of follow-up; 3) the availability of preoperative, immediately postoperative, and latest follow-up full-length standing radiographs; and 4) completed HRQOL questionnaires at the baseline and latest follow-up. Patients were excluded if they had undergone a PSO performed through pseudarthrosis or a total hip arthroplasty (THA). Considering that sagittal realignment failure was defined as a postoperative sagittal vertical axis (SVA) $>80 \mathrm{~mm}$, patients were grouped according to the postoperative SVA measurement. 8,15 Those with an immediately postoperative SVA $<80 \mathrm{~mm}$ and an SVA $<80 \mathrm{~mm}$ at the latest follow-up were classified as having achieved successful realignment (group A). Patients with an immediately postoperative SVA $>80 \mathrm{~mm}$ were classified as having inadequate correction (group B), and those patients with an immediately postoperative SVA $<80 \mathrm{~mm}$ but an SVA $>$ $80 \mathrm{~mm}$ at the latest follow-up were classified as having sagittal decompensation during follow-up (group C).

Surgical decisions were made mainly based on lateral full-length radiographs. The amount of correction and the osteotomy level were designed with the aim of restoring a balanced standing posture and horizontal gaze. The osteotomy level was principally selected at the apex vertebra. Closing wedge osteotomy was recommended for patients with a required amount of correction $<35^{\circ}$. When the amount of correction was between $35^{\circ}$ and $60^{\circ}$, a closingopening wedge osteotomy was performed. Moreover, a second PSO was considered for patients with a required amount of correction $>60^{\circ}$. Intraoperative fluoroscopy was performed to confirm the obtained correction magnitude. The instrumented fusion routinely covered 4 levels above and 3 levels below the PSO level. For patients with continuous multilevel ALL ossification that distally ended at 1 or 2 levels cephalad to the initial UIV, the fusion was proximally extended to the level with ALL ossification. Moreover, fusion was extended to the sacrum when the initial LIV could not provide adequate corrective force and biomechanical stability. Furthermore, fusion to the pelvis was considered in the following cases: 1) severe osteoporosis, 2) high BMI, 3) high disease activity with absent sacroiliac joint autofusion, 4) combined sagittal and coronal plane deformity, 5) pseudarthrosis at the lumbosacral region (L5S1), and 6) a PSO level selected at the lumbosacral region. Postoperative bracing was routinely applied for 3 months.

\section{Radiological Evaluation}

Radiographs were analyzed using Surgimap software (Nemaris, Inc.). Radiographic parameters were measured on full-length standing lateral radiographs and included thoracic kyphosis (TK), lumbar lordosis (LL), SVA, pelvic incidence (PI), sacral slope (SS), pelvic tilt (PT), and osteotomy angle (OVA) (Fig. 1). ${ }^{16}$ The PI-LL mismatch was also calculated. Additionally, the proximal junctional angle (PJA) and the distal junctional angle (DJA) were measured. ${ }^{17,18}$ The DJA was not measured in patients with LIV at or below S1. A positive angle indicates kyphosis.

Structural damage of the bilateral hip joints was assessed according to the Bath Ankylosing Spondylitis Radiology Index of the hip (BASRI-h).${ }^{19} \mathrm{~A}$ higher grade of the BASRI-h was adopted for further statistical analysis. Ossification of the ALL at the proximal junction (lower endplate of the UIV to the upper endplate of the two supra-adjacent vertebrae), PSO level, and distal junction 

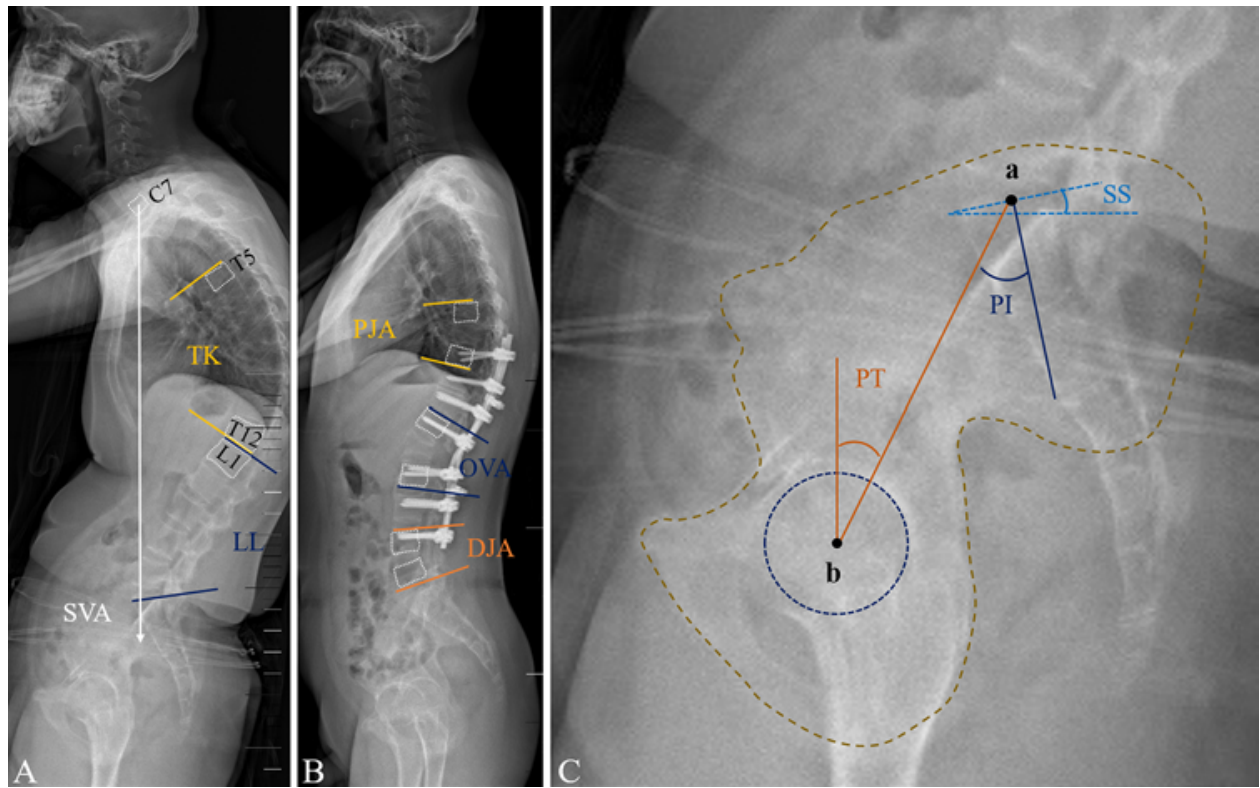

FIG. 1. Radiographic measurements of spinopelvic parameters. A: TK, T5-12 Cobb angle; LL, L1-S1 Cobb angle; and SVA, the horizontal distance between the $\mathrm{C} 7$ plumb line and the posterior superior corner of S1. B: PJA, the Cobb angle between UIV and $\mathrm{UIV}+2$; OVA, the Cobb angle between the osteotomy vertebra-1 and the osteotomy vertebra+1; and DJA, the Cobb angle between LIV and LIV+1. C: PT, the angle between the vertical and the line drawn from point a to the femoral head axis (point b); SS, the angle between the sacral plate and the horizontal; and $\mathrm{PI}$, the angle between the line from point $a$ to point $b$ and perpendicular to the sacral plate.

(upper endplate of the LIV to the lower endplate of the one infra-adjacent vertebra) were evaluated according to the modified Stoke Ankylosing Spondylitis Spine Score (mSASSS). ${ }^{20}$ Adequate ALL ossification was defined as formation of a complete bony bridge between the upper and lower vertebral edges.

\section{Clinical Evaluation}

Demographic data, including sex, age, and follow-up duration, were collected as well as surgical data including the osteotomy level and fusion levels. Intraoperative sagittal translation (ST) was defined as the translation of $>5 \mathrm{~mm}$ between the posterior inferior edge of the cranial vertebra and the posterior superior edge of the caudal vertebra at the PSO level. ${ }^{21}$ Mechanical complications during follow-up including RF, proximal junctional kyphosis (PJK), distal junctional kyphosis (DJK), and pseudarthrosis were also reviewed. PJK and DJK were defined as a PJA or DJA $>10^{\circ}$ and at least $10^{\circ}$ larger than the preoperative measurement, respectively. ${ }^{17,18}$ Pseudarthrosis at the PSO level was determined by RF at the PSO level, progression of deformity, and apparent enhanced sclerosis surrounding the osteotomy vertebral endplates. ${ }^{22} \mathrm{HRQOL}$ questionnaires included the Oswestry Disability Index (ODI) and visual analog scale (VAS) for back pain.

\section{Statistical Analysis}

Data analyses were performed using SPSS version 18 (SPSS Inc.). Variables in groups B and C were compared with those in group A. The Student t-test was used to assess differences in continuous variables and the chisquare test for differences in categorical variables. More- over, differences in categorical variables with fewer than 5 observations were analyzed using the Fisher's exact test; $\mathrm{p}<0.05$ was considered statistically significant. Variables with $\mathrm{p}<0.05$ were further included in the stepwise logistic analysis. Variables with $\mathrm{p}<0.05$ identified as the independent factors. Receiver operating characteristic curves were further used to determine the cutoff values of variables for sagittal reconstruction failure.

\section{Results}

A total of 109 patients with a mean age of 35.3 years (range 18-66 years) were included in the current study. The mean follow-up was 3.2 years (range 2-8 years). There were 80 patients $(73.4 \%)$ in group A, $16(14.7 \%)$ in group B, and 13 (11.9\%) in group C. Demographic and surgical data are summarized in Table 1. Compared with group A, group B and group C patients were older (mean ages 43.6 vs 32.9 years, $p<0.001$; and 39.2 vs 32.9 years, $\mathrm{p}=0.018$, respectively). There was a higher proportion of patients with adequate ALL ossification at the PSO level in group B than in group A $(37.5 \%$ vs $22.5 \%, \mathrm{p}=0.003)$.

Preoperative LL, PT, SVA, and PI-LL were larger in group B ( $p<0.05$ for all) (Table 2). There were no significant differences between groups $\mathrm{A}$ and $\mathrm{B}$ in postoperative changes of any radiographic parameters. However, the DJA at the latest follow-up was larger in group B than in group A $\left(-8.6^{\circ} \mathrm{vs}-14.8^{\circ}, \mathrm{p}=0.033\right)$. The preoperative SVA was larger in group C than in group A (176.0 vs 139.6 $\mathrm{mm}, \mathrm{p}=0.040$ ) (Table 2). No significant differences were found between groups $\mathrm{A}$ and $\mathrm{C}$ in postoperative changes of the OVA, LL, or SVA. During follow-up, changes in the OVA, LL, and SVA were significantly greater in group C 
TABLE 1. Comparison of demographic, surgical, and complications data

\begin{tabular}{|c|c|c|c|c|c|}
\hline & Group A $(n=80)$ & Group B $(n=16)$ & p Value* & Group C $(n=13)$ & p Value $†$ \\
\hline Male sex & $72(90.1)$ & $14(87.5)$ & $>0.99$ & $13(100)$ & 0.594 \\
\hline Mean age, yrs & $32.9 \pm 8.4$ & $43.6 \pm 12.1$ & $<0.001$ & $39.2 \pm 11.2$ & 0.018 \\
\hline Mean follow-up, yrs & $3.0 \pm 1.3$ & $3.2 \pm 1.8$ & 0.630 & $4.0 \pm 1.7$ & 0.017 \\
\hline Mean fusion levels & $8.8 \pm 1.1$ & $8.8 \pm 1.2$ & 0.937 & $8.6 \pm 1.3$ & 0.621 \\
\hline PSO level & & & $<0.001$ & & 0.004 \\
\hline L1 & $18(22.5)$ & $2(12.5)$ & & $1(7.7)$ & \\
\hline L2 & $58(72.5)$ & $7(43.8)$ & & $7(53.8)$ & \\
\hline L3 & $4(5.0)$ & $7(43.8)$ & & $5(38.5)$ & \\
\hline \multicolumn{6}{|l|}{ BASRI-h grade 4} \\
\hline Preop & 0 & $2(12.5)$ & 0.026 & 0 & NA \\
\hline Latest follow-up & 0 & $3(18.8)$ & 0.004 & $2(15.4)$ & 0.018 \\
\hline ALL ossification & $18(22.5)$ & $6(37.5)$ & 0.003 & $3(23.1)$ & 0.604 \\
\hline Mean EBL, ml & $1945.6 \pm 890.6$ & $1803.1 \pm 979.2$ & 0.567 & $2273.1 \pm 1173.8$ & 0.243 \\
\hline Dural tear & $3(3.8)$ & $1(6.3)$ & 0.524 & $1(7.7)$ & 0.458 \\
\hline Postural brachial plexus palsy & $2(2.5)$ & 0 & $>0.99$ & $1(7.7)$ & 0.367 \\
\hline Motor deficit & $1(1.3)$ & $1(6.3)$ & 0.307 & 0 & $>0.99$ \\
\hline Transient radiculopathy & $2(2.5)$ & 0 & $>0.99$ & $1(7.7)$ & 0.367 \\
\hline Sagittal translation & $5(6.3)$ & $2(12.5)$ & 0.330 & $1(7.7)$ & $>0.99$ \\
\hline RF & $7(8.8)$ & $2(12.5)$ & 0.642 & $5(38.5)$ & 0.011 \\
\hline Pseudarthrosis & 0 & 0 & NA & $2(15.4)$ & 0.018 \\
\hline PJK & $7(8.8)$ & $1(6.3)$ & $>0.99$ & $1(7.7)$ & $>0.99$ \\
\hline DJK & 0 & $1(6.3)$ & 0.167 & 0 & NA \\
\hline
\end{tabular}

$\mathrm{EBL}=$ estimated blood loss; $\mathrm{NA}=$ not applicable.

Values represent the number of patients (\%) or mean \pm SD unless indicated otherwise.

${ }^{*}$ Comparison between group $A$ and group $B$.

$\dagger$ Comparison between group $A$ and group $C$

( $\mathrm{p}<0.05$ for all). However, no differences were found in changes of TK, PT, SS, PJA, or DJA between groups A and $\mathrm{C}$ during follow-up.

Stepwise logistic regression analysis identified age and preoperative PT and SVA as independent risk factors for immediate postoperative inadequate correction (Table 3). For sagittal decompensation, age and preoperative SVA were identified as independent risk factors. Furthermore, cutoff values for inadequate correction were an age $>37.5$ years, PT $>42.5^{\circ}$, and SVA $>189.0 \mathrm{~mm}$. Additionally, thresholds for sagittal decompensation were an age $>40.5$ years and SVA > $169.5 \mathrm{~mm}$.

\section{Complications}

Dural tear, postural brachial plexus palsy, motor deficit, transient radiculopathy, and sagittal translation were observed in the current study (Table 1). Additionally, PJK developed in 7 patients in group A, 1 in group B, and 1 in group $\mathrm{C}$. There was no difference regarding the incidence of PJK between the 3 groups. One patient in group B developed DJK with SVA progression from $92 \mathrm{~mm}$ immediately after surgery to $140 \mathrm{~mm}$ at the latest followup. However, DJK was not found in group A or group B. A higher incidence of RF was found in group $C$ than in group A $(38.5 \%$ vs $8.8 \%, \mathrm{p}=0.011)$. There were 2 patients with $\mathrm{RF}$ accompanied by pseudarthrosis in group $\mathrm{C}$, and the rate of pseudarthrosis in group $\mathrm{C}$ was higher than in group A $(15.4 \%$ vs $0, p=0.018)$. A higher distribution of patients with a BASRI-h grade 4 was found in group B than in group A preoperatively $(12.5 \%$ vs $0, p=0.026)$. At the latest follow-up, a higher distribution of patients with a BASRI-h grade progressing to grade 4 were also found in group $\mathrm{C}$ than in group $\mathrm{A}(15.4 \%$ vs $0, \mathrm{p}=0.018)$.

\section{Subanalysis of the Effect of ALL Ossification on Development of Mechanical Complications During Follow-Up}

At the latest follow-up, 47 patients presented with adequate ALL ossification at the proximal junction. Meanwhile, adequate ALL ossification at the distal junction was found in 36 patients; 21 patients with LIV at S1 were not included in this subanalysis (Table 4). Changes in the PJA $\left(0.5^{\circ}\right.$ vs $\left.2.2^{\circ}, \mathrm{p}=0.027\right)$ and DJA $\left(0.3^{\circ}\right.$ vs $\left.2.2^{\circ}, \mathrm{p}=0.019\right)$ were less in patients with adequate ALL ossification at the proximal and distal junctions, respectively. A higher incidence of RF was found in patients without adequate ossification of ALL (19.6\% vs 6.9\%, $\mathrm{p}=0.045)$. The mean increase of SVA during follow-up was $32.8 \mathrm{~mm}$ in all 14 patients with RF. In detail, the mean increase of SVA was $81.0 \mathrm{~mm}$ in the 2 patients with RF accompanied by pseudarthrosis, and $18.0 \mathrm{~mm}$ in the 4 patients with RF and adequate ALL ossification at the PSO level. 
TABLE 2. Comparison of the preoperative, immediately postoperative, and latest follow-up radiographic parameters

\begin{tabular}{|c|c|c|c|c|c|}
\hline & Group A $(n=80)$ & Group B $(n=16)$ & p Value* & Group C $(n=13)$ & $\mathrm{p}$ Value $†$ \\
\hline \multicolumn{6}{|l|}{$\mathrm{TK}^{\circ}{ }^{\circ}$} \\
\hline Preop & $44.3 \pm 17.7$ & $53.0 \pm 11.3$ & 0.062 & $47.3 \pm 16.8$ & 0.568 \\
\hline Postop & $43.3 \pm 13.4$ & $51.3 \pm 8.9$ & 0.026 & $45.0 \pm 14.8$ & 0.678 \\
\hline Latest follow-up & $48.1 \pm 13.6 \ddagger$ & $55.4 \pm 11.5 \ddagger$ & 0.050 & $50.7 \pm 13.0 \ddagger$ & 0.528 \\
\hline \multicolumn{6}{|l|}{$\mathrm{LL},{ }^{\circ}$} \\
\hline Preop & $-9.6 \pm 19.1$ & $1.0 \pm 19.7$ & 0.047 & $-4.1 \pm 17.3$ & 0.330 \\
\hline Postop & $-54.5 \pm 14.0 \S$ & $-44.8 \pm 10.6 \S$ & 0.010 & $-48.1 \pm 10.6 \S$ & 0.115 \\
\hline Latest follow-up & $-50.8 \pm 14.2 \ddagger$ & $-41.3 \pm 10.4$ & 0.014 & $-39.6 \pm 15.5 \ddagger$ & 0.011 \\
\hline \multicolumn{6}{|l|}{ SVA, mm } \\
\hline Preop & $139.6 \pm 58.0$ & $200.7 \pm 52.7$ & $<0.001$ & $176.0 \pm 60.7$ & 0.040 \\
\hline Postop & $22.4 \pm 32.8 \S$ & $100.6 \pm 17.8 \S$ & $<0.001$ & $31.3 \pm 39.6 \S$ & 0.381 \\
\hline Latest follow-up & $23.1 \pm 32.4$ & $104.1 \pm 24.6$ & $<0.001$ & $99.5 \pm 19.8 \ddagger$ & $<0.001$ \\
\hline \multicolumn{6}{|l|}{$\mathrm{PI},{ }^{\circ}$} \\
\hline Preop & $44.6 \pm 10.8$ & $50.9 \pm 10.5$ & 0.035 & $47.2 \pm 11.5$ & 0.415 \\
\hline Postop & $44.3 \pm 10.9$ & $49.4 \pm 9.3$ & 0.086 & $44.6 \pm 10.5 \S$ & 0.926 \\
\hline Latest follow-up & $44.6 \pm 10.1$ & $50.5 \pm 9.2$ & 0.034 & $46.0 \pm 10.6$ & 0.653 \\
\hline \multicolumn{6}{|l|}{$S S,{ }^{\circ}$} \\
\hline Preop & $9.5 \pm 9.3$ & $7.3 \pm 8.9$ & 0.394 & $9.1 \pm 8.7$ & 0.893 \\
\hline Postop & $27.2 \pm 9.4 \S$ & $22.9 \pm 8.6 \S$ & 0.091 & $21.5 \pm 5.5 \S$ & 0.038 \\
\hline Latest follow-up & $23.0 \pm 8.9 \ddagger$ & $19.9 \pm 7.8$ & 0.193 & $21.5 \pm 12.5$ & 0.603 \\
\hline \multicolumn{6}{|l|}{$\mathrm{PT},{ }^{\circ}$} \\
\hline Preop & $35.0 \pm 8.5$ & $43.6 \pm 10.8$ & 0.001 & $38.1 \pm 8.4$ & 0.234 \\
\hline Postop & $16.8 \pm 8.3 \S$ & $26.5 \pm 7.3 \S$ & $<0.001$ & $23.1 \pm 10.2 \S$ & 0.017 \\
\hline Latest follow-up & $21.6 \pm 9.0 \ddagger$ & $30.6 \pm 10.9$ & 0.001 & $25.2 \pm 8.7$ & 0.181 \\
\hline \multicolumn{6}{|l|}{ OVA, ${ }^{\circ}$} \\
\hline Preop & $15.5 \pm 11.3$ & $14.4 \pm 13.4$ & 0.740 & $10.3 \pm 10.5$ & 0.125 \\
\hline Postop & $-29.4 \pm 8.3 \S$ & $-32.2 \pm 8.7 \S$ & 0.230 & $-31.4 \pm 6.9 \S$ & 0.421 \\
\hline Latest follow-up & $-28.9 \pm 8.9$ & $-30.1 \pm 8.1$ & 0.620 & $-26.1 \pm 10.6 \ddagger$ & 0.302 \\
\hline \multicolumn{6}{|l|}{ PI-LL, ${ }^{\circ}$} \\
\hline Preop & $35.0 \pm 16.1$ & $51.9 \pm 19.5$ & $<0.001$ & $43.2 \pm 16.9$ & 0.095 \\
\hline Postop & $-10.2 \pm 11.9 \S$ & $4.6 \pm 8.4 \S$ & $<0.001$ & $-3.5 \pm 12.0 \S$ & 0.061 \\
\hline Latest follow-up & $-6.1 \pm 12.8 \ddagger$ & $9.2 \pm 11.4$ & $<0.001$ & $6.4 \pm 13.2 \ddagger$ & 0.002 \\
\hline \multicolumn{6}{|l|}{ PJA, ${ }^{\circ}$} \\
\hline Preop & $12.0 \pm 7.2$ & $15.7 \pm 7.5$ & 0.064 & $11.4 \pm 5.9$ & 0.779 \\
\hline Postop & $12.5 \pm 6.6$ & $16.0 \pm 6.0$ & 0.053 & $12.7 \pm 6.2$ & 0.917 \\
\hline Latest follow-up & $14.0 \pm 6.1 \ddagger$ & $16.5 \pm 8.1$ & 0.166 & $14.9 \pm 6.8$ & 0.660 \\
\hline \multicolumn{6}{|l|}{ DJA, ${ }^{\circ}$} \\
\hline Preop & $-17.3 \pm 7.4$ & $-12.3 \pm 5.4$ & 0.056 & $-15.2 \pm 5.5$ & 0.423 \\
\hline Postop & $-15.9 \pm 7.5 \S$ & $-12.2 \pm 5.9$ & 0.164 & $-13.9 \pm 4.7 \S$ & 0.443 \\
\hline Latest follow-up & $-14.8 \pm 7.9 \ddagger$ & $-8.6 \pm 9.7$ & 0.033 & $-12.7 \pm 5.0 \ddagger$ & 0.443 \\
\hline
\end{tabular}

Values are presented as mean \pm SD unless indicated otherwise.

* Comparison between the values in group $A$ and group $B$.

† Comparison between the values in group $A$ and group $C$.

$\ddagger$ Statistically significant between the immediate postoperative value and the latest follow-up value.

$\S$ Statistically significant between the baseline value and the immediate postoperative value.

In contrast to the improved ODI scores in group A, improvement in ODI scores was not found in groups $\mathrm{B}$ or $\mathrm{C}$ at the latest follow-up (Table 5). Eight patients developed intraoperative ST, 4 of whom had adequate ALL ossification at the PSO level. Revision surgery was performed in 7 patients with RF (1 of whom had concurrent pseudarthrosis and PJK). Generally, broken rods were replaced with new intact rods and the satellite rods across the PSO level were also used in the revision surgery. For the patient with pseudarthrosis accompanied by PJK, a T12 PSO was 
TABLE 3. Multivariate analysis of variables associated with sagittal reconstruction failure

\begin{tabular}{lccc}
\hline & OR & $95 \% \mathrm{Cl}$ & $\mathrm{p} \mathrm{Value}$ \\
\hline Inadequate correction & & & \\
\hline Age & 1.102 & $1.023-1.188$ & 0.011 \\
\hline SVA & 1.041 & $1.008-1.076$ & 0.016 \\
\hline PT & 1.171 & $1.006-1.363$ & 0.041 \\
\hline Sagittal decompensation & & & \\
\hline Age & 1.101 & $1.023-1.186$ & 0.011 \\
\hline SVA & 1.013 & $1.002-1.024$ & 0.020 \\
\hline
\end{tabular}

also performed. No permanent neurological complications were identified.

\section{Discussion}

The role of sagittal alignment for maintaining an energy-efficient posture has been well recognized. ${ }^{8}$ Despite the improved sagittal alignment, reconstruction failure after PSO has frequently been reported in ASD. ${ }^{3,6,8-10}$ Reconstruction failure could be attributed to inadequate correction immediately after surgery and sagittal decompensation caused by mechanical complications, including RF, PJK, and DJK, during follow-up. ${ }^{6,8-10}$ However, due to heterotopic ossification, the rates and patterns of sagittal reconstruction failure following PSO might be different in AS-related rigid thoracolumbar kyphosis.

\section{Inadequate Correction Immediately After Surgery}

In line with a previous study, older age, and greater preoperative PT and SVA were identified as the independent risk factors of inadequate correction in the current study. ${ }^{8}$ Furthermore, the cutoff values were an age $>37.5$ years, PT $>42.5^{\circ}$, and SVA $>189.0 \mathrm{~mm}$. Theoretically, achieving adequate sagittal realignment would require a greater magnitude of correction in group B than in group A. However, there was no significant difference in the magnitude of deformity correction between the two groups. Therefore, patients in group B developed inadequate correction due to the required correction not being obtained.

Inadequate correction could be caused by patient-related factors and surgical factors. A larger amount of correction might be accompanied by a prolonged surgery duration, increased blood loss, and higher risk of complications. ${ }^{17}$ Therefore, in the older patient, the required magnitude of correction might not be performed as vigorously as that in the younger patient. Additionally, PSO was a technically demanding surgery, especially in rigid AS-related thoracolumbar kyphosis. Any mistakes in preoperative osteotomy planning or intraoperative manipulation might result in inadequate correction. Full-balance integrated index could serve as a useful osteotomy planning tool, especially in AS-related thoracolumbar kyphosis with compensated knee flexion. ${ }^{23}$ Moreover, adequate ALL ossification at the PSO level was identified as a risk factor of inadequate correction in the current study. During the closure of the osteotomy gap, the lordosing effect of the neighboring disc opening might be impaired by adequate ALL ossifi-
TABLE 4. Effect of adequate ALL ossification on the development of mechanical complications

\begin{tabular}{|c|c|c|c|}
\hline & $\begin{array}{c}\text { w/o Adequate } \\
\text { ALL Ossification }\end{array}$ & $\begin{array}{c}\text { w/ Adequate } \\
\text { ALL Ossification }\end{array}$ & $\begin{array}{c}p \\
\text { Value }\end{array}$ \\
\hline \multicolumn{4}{|l|}{ Proximal junction } \\
\hline No. of pts & 62 & 47 & \\
\hline Mean PJA change, ${ }^{\circ}$ & $2.2 \pm 4.5$ & $0.5 \pm 3.3$ & 0.027 \\
\hline \multicolumn{4}{|l|}{ Distal junction } \\
\hline No. of pts & 52 & 36 & \\
\hline Mean DJA change, ${ }^{\circ}$ & $2.2 \pm 4.8$ & $0.3 \pm 2.4$ & 0.019 \\
\hline \multicolumn{4}{|l|}{ PSO level } \\
\hline No. of pts & 51 & 58 & \\
\hline RF & $10(19.6)$ & $4(6.9)$ & 0.045 \\
\hline Pseudarthrosis & $2(3.9)$ & 0 & 0.217 \\
\hline
\end{tabular}

cation at the PSO level. ${ }^{14}$ However, this impairment of the lordosing effect could be partly compensated for by the intraoperative ST. As previously reported, a comparable magnitude of deformity correction could be obtained with a few millimeters of ST. ${ }^{21}$ Notably, purposeful ST should be avoided because of the potential neurological complications. In these patients with adequate ALL ossification at the PSO level, a two-level PSO might be considered to obtain adequate correction.

At the latest follow-up, the DJA was larger in group $\mathrm{B}$; additionally, there was 1 patient who developed DJK in group B. As previously reported, in ASD after undercorrection, greater stress would be expected at the distal junction as a result of the anteriorly moved gravity center. Furthermore, prolapse of the distal junction discs was more common in younger patients without degeneration of the distal junction discs. ${ }^{24}$ This phenomenon was in line with our results: changes in the junctional angle during follow-up were greater in patients without adequate ALL ossification. In patients with postoperative malalignment, ossification of the ALL along with an ossified disc at the distal junction might provide a more stable base for fixation that could sustain increased stress. Consequently, a more severe sagittal malalignment would be expected after occurrence of DJK. In our experience, besides the ideal postoperative sagittal realignment, selecting the LIV at a level with adequate ALL ossification, or at the sacrum, might be practical to decrease the occurrence of DJK.

\section{Sagittal Decompensation During Follow-Up}

Older age and greater preoperative SVA were identified as the independent risk factors of sagittal decompensation during follow-up. Moreover, the cutoff values were an age $>40.5$ years and SVA $>169.5 \mathrm{~mm}$. Of the 4 mechanical complications encountered, including PJK, DJK, RF, and pseudarthrosis, only RF and pseudarthrosis were found to be associated with sagittal decompensation. Loss of correction within the fusion levels was mostly caused by pseudarthrosis. ${ }^{10}$ In ASD, pseudarthrosis was highly associated 
TABLE 5. Comparison of clinical outcomes

\begin{tabular}{|c|c|c|c|c|c|c|}
\hline & \multicolumn{3}{|c|}{ ODI } & \multicolumn{3}{|c|}{ VAS } \\
\hline & Preop & Latest Follow-Up & $p$ Value & Preop & Latest Follow-Up & $p$ Value \\
\hline Group A $(n=80)$ & $28.9 \pm 18.4$ & $14.8 \pm 13.3$ & $<0.001$ & $4.5 \pm 2.7$ & $2.1 \pm 2.0$ & $<0.001$ \\
\hline Group B $(n=16)$ & $26.4 \pm 16.4$ & $20.5 \pm 16.8$ & 0.147 & $4.3 \pm 2.4$ & $2.3 \pm 2.2$ & 0.015 \\
\hline Group C ( $n=13)$ & $26.2 \pm 15.9$ & $17.2 \pm 13.9$ & 0.171 & $4.4 \pm 2.8$ & $2.2 \pm 1.8$ & 0.044 \\
\hline
\end{tabular}

Values are presented as mean \pm SD unless indicated otherwise.

with RF. However, due to the superior bone fusion capacity in AS, pseudarthrosis developed less frequently following PSO.$^{25}$ In contrast, formation of adequate ALL ossification at the PSO level might protect rods from fracture. After $\mathrm{RF}$, large mechanical stress would be translated from the rod to the posterior columns, and the posterior elements might be incapable of resisting the resultant tensile forces. Consequently, the $\mathrm{C} 7$ plumb line would move anteriorly.

In addition, the increase of the SVA in patients with pseudarthrosis was larger than that in all patients with RF (81 mm vs $32 \mathrm{~mm}$ ). Occurrence of pseudarthrosis could be ascribed to the intraoperative wide resection of the posterior bony elements. Failure of the fusion process was expected due to the resultant instability and insufficient bony bed. Sagittal malalignment after RF would be further deteriorated by pseudarthrosis (Fig. 2). Notably, no patients with RF and adequate ALL ossification at the PSO level developed sagittal decompensation. Adequate ossification of the ALL could be reasonably inferred as the anterior support that counteracted the anteriorly moved gravity center. For these patients, RF might not directly lead to sagittal malalignment recurrence. In clinical practice, a lower rod contour angle at the PSO level and application of satellite rods would protect the rods from fracture. ${ }^{25}$

In the current study, PJK was not considered as the reason for sagittal decompensation due to the ankylosing spine. Different from the rapid and angular change in ASD, most PJK in AS presented with loss of disc space height and mild and uniform squaring of the vertebral bodies. However, it was reasonable to anticipate that sagittal decompensation might be found when PJK deterioration occurred with the increasing follow-up period. ${ }^{26}$ Additionally, the junctional region with adequate ALL ossification was more solid to support the increased junctional stress concentration, thereby relieving the junctional kyphotic changes. Thus, selection of UIV at a level with adequate ALL ossification might help decrease the incidence of PJK.

Compared with proximal unfused segments, kyphotic change in distal unfused segments is more likely to cause sagittal imbalance due to the longer moment arm. ${ }^{15}$ However, increased change in the DJA was not found in group C. Instead, a progression to BASRI-h grade 4 was consid-
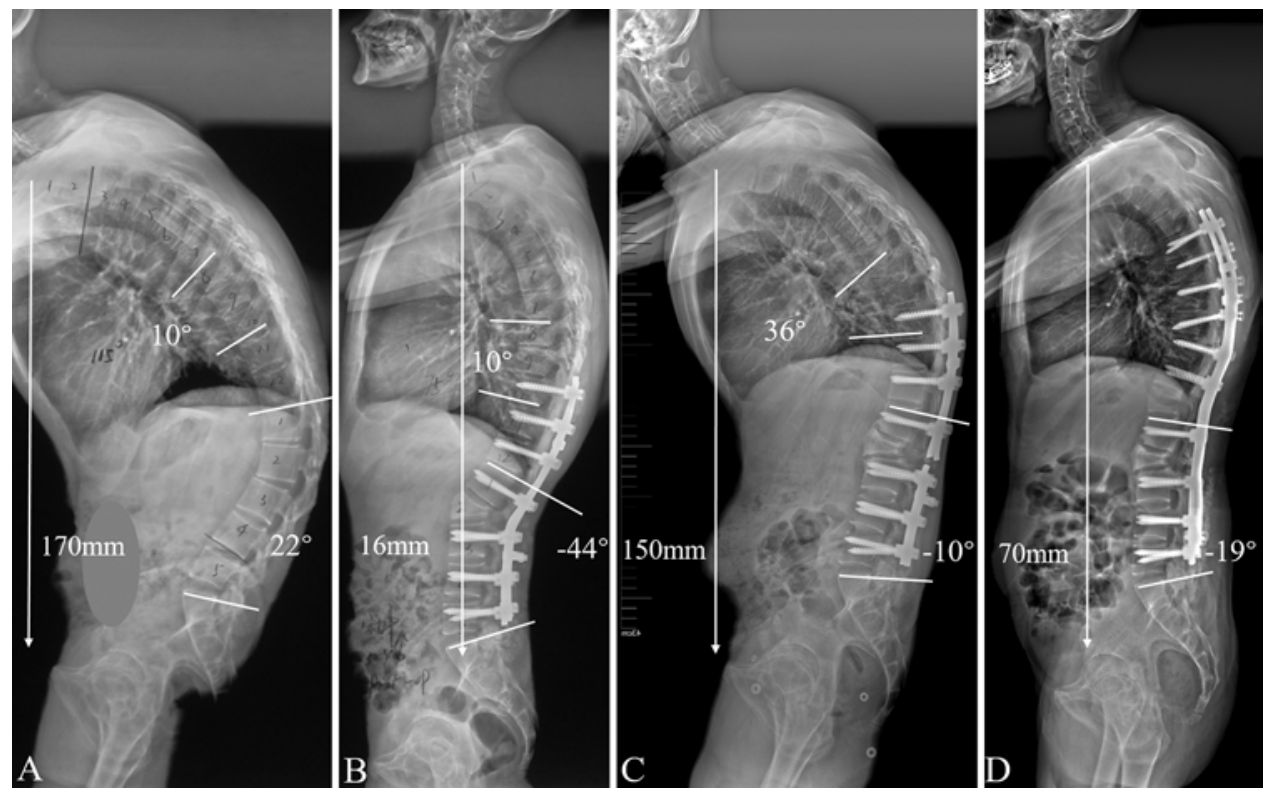

FIG. 2. Lateral radiographs obtained in a 32-year-old male patient with $A S$ and thoracolumbar kyphosis who underwent an $L 2$ PSO. A: Before the PSO, the LL and SVA were $22^{\circ}$ and $170 \mathrm{~mm}$, respectively. B: After the PSO, the LL was improved to $-44^{\circ}$ with the SVA decreased to $16 \mathrm{~mm}$. C: At the 7-year follow-up, the PJA was increased from $10^{\circ}$ preoperatively to $36^{\circ}$, rod fracture accompanied by pseudarthrosis at the PSO level was found, and the SVA was increased to $150 \mathrm{~mm}$. D: A T12 PSO was performed, and the broken rods were replaced at the revision surgery. Satellite rods were also applied across the PSO levels. SVA was decreased to $70 \mathrm{~mm}$ after revision surgery. 

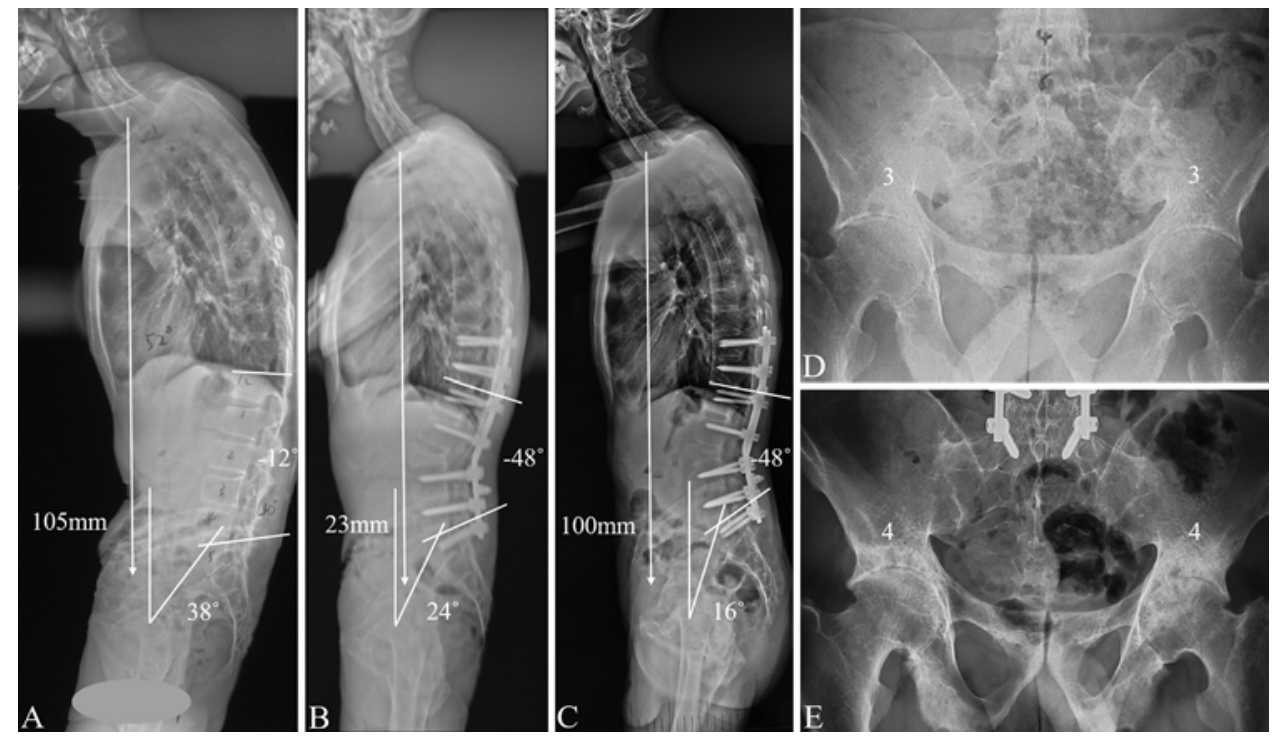

FIG. 3. Lateral radiographs obtained in a 38-year-old male patient with AS and thoracolumbar kyphosis following an L3 PSO. A: Before the PSO, PT and SVA were $38^{\circ}$ and $105 \mathrm{~mm}$, respectively. B: After the PSO, LL was improved from $-12^{\circ}$ to $-48^{\circ}$. The PT and SVA were decreased to $24^{\circ}$ and $23 \mathrm{~mm}$, respectively. C: At the 4-year follow-up, TK and LL remained unchanged. The PT was decreased to $16^{\circ}$ with the SVA deteriorated to $100 \mathrm{~mm}$. D and E: Hip structural damage deteriorated, with a BASRI-h grade 3 immediately after the PSO to a grade 4 at the 4 -year follow-up.

ered as another reason for sagittal decompensation (Fig. 3). Forward pelvic rotation occurred with the progression of hip structural damage. ${ }^{27}$ The effect of pelvic forward rotation on spinal sagittal alignment was similar to that found in DJK. Moreover, the moment arm was even longer at the hip joint than at the distal junction. Therefore, hip joint function was essential for maintaining sagittal balance. ${ }^{27}$ Medication or even THA should be recommended for patients with progressive hip structural damage.

At the latest follow-up, the ODI score was significantly improved in group A due to the improved sagittal balance. However, significantly improved ODI scores at the latest follow-up were not found in group B or group C, indicating that restoration and maintenance of sagittal alignment was of great importance for the physiological function of patients with AS. The need for revision surgery should be guided by symptoms and radiographic changes. Revision was recommended only for patients with severe sagittal malalignment and unrelieved pain after conservative treatment. 25

Several limitations of the current study should be addressed. First, it was retrospectively designed. Second, due to the higher radiation dose of CT, fusion status at the PSO level was evaluated using radiography Moreover, fusion status was confirmed through surgical exploration in the patients who underwent revision surgery. Third, sagittal alignment would be theoretically restored after THA due to improved hip function; however, the improvement of sagittal alignment after THA in patients with hip structural damage was not confirmed in the current study. Future study with a larger patient sample is needed to specifically investigate the spinal alignment change following THA. Finally, a 2-year follow-up might be too short to fully analyze the sagittal alignment change in AS. The junctional kyphotic change and RF rate might increase during longer follow-up, resulting in patients in group A being relocated to group C; therefore, a longer period of follow-up is needed to investigate the rates of sagittal decompensation.

\section{Conclusions}

Sagittal reconstruction failure in AS-related thoracolumbar kyphosis following PSO included inadequate correction (14.7\%) and sagittal decompensation (11.9\%). RF was the most common factor for sagittal decompensation. Adequate ALL ossification was a risk factor of inadequate correction. However, formation of adequate ALL ossification during follow-up would decrease the RF rate and relieve the junctional kyphotic changes. Older age and greater baseline SVA were independent risk factors for both inadequate correction and sagittal decompensation. Notably, severe hip structural damage would also lead to sagittal imbalance.

\section{Acknowledgments}

B.P.Q. has received funding from Jiangsu Provincial Medical Talent Program (ZDRCA2016068). Y.Q. has received funding from the Jiangsu Provincial Key Medical Center (YXZXA2016009).

\section{References}

1. Braun J, Sieper J. Ankylosing spondylitis. Lancet. 2007; 369(9570):1379-1390.

2. Zhao SZ, Qian BP, Qiu Y, Wang B, Huang JC, Qiao M. Impact of cervical range of motion on the global spinal alignment in ankylosing spondylitis patients with thoracolumbar kyphosis following pedicle subtraction osteotomy. Spine J. 2020;20(2):241-250.

3. Huang JC, Qian BP, Qiu Y, Wang B, Yu Y, Qiao M. What is the optimal postoperative sagittal alignment in ankylosing 
spondylitis patients with thoracolumbar kyphosis following one-level pedicle subtraction osteotomy? Spine J. 2020;20(5): 765-775.

4. Lee KH, Kim KT, Kim YC, Lee JW, Ha KY. Radiographic findings for surgery-related complications after pedicle subtraction osteotomy for thoracolumbar kyphosis in 230 patients with ankylosing spondylitis. J Neurosurg Spine. 2020; 33(3):366-372.

5. Qiao M, Qian BP, Mao SH, Qiu Y, Wang B. The patterns of loss of correction after posterior wedge osteotomy in ankylosing spondylitis-related thoracolumbar kyphosis: a minimum of five-year follow-up. BMC Musculoskelet Disord. 2017;18(1):465.

6. Kim YJ, Bridwell KH, Lenke LG, Cheh G, Baldus C. Results of lumbar pedicle subtraction osteotomies for fixed sagittal imbalance: a minimum 5-year follow-up study. Spine (Phila Pa 1976). 2007;32(20):2189-2197.

7. Rose PS, Bridwell KH, Lenke LG, Cronen GA, Mulconrey DS, Buchowski JM, Kim YJ. Role of pelvic incidence, thoracic kyphosis, and patient factors on sagittal plane correction following pedicle subtraction osteotomy. Spine (Phila $\mathrm{Pa}$ 1976). 2009;34(8):785-791.

8. Schwab FJ, Patel A, Shaffrey CI, Smith JS, Farcy JP, Boachie-Adjei O, et al. Sagittal realignment failures following pedicle subtraction osteotomy surgery: are we doing enough? J Neurosurg Spine. 2012;16(6):539-546.

9. Lee SH, Kim KT, Suk KS, Lee JH, Seo EM, Huh DS. Sagittal decompensation after corrective osteotomy for lumbar degenerative kyphosis: classification and risk factors. Spine (Phila Pa 1976). 2011;36(8):E538-E544.

10. Eichler D, Charles YP, Baldairon F, Ntilikina Y, Sauleau EA, Steib JP. Possible factors associated with sagittal malalignment recurrence after pedicle subtraction osteotomy. Eur Spine J. 2019;28(1):161-169.

11. Arlet V, Aebi M. Junctional spinal disorders in operated adult spinal deformities: present understanding and future perspectives. Eur Spine J. 2013;22(suppl 2):S276-S295.

12. Cho KJ, Lenke LG, Bridwell KH, Kamiya M, Sides B. Selection of the optimal distal fusion level in posterior instrumentation and fusion for thoracic hyperkyphosis: the sagittal stable vertebra concept. Spine (Phila Pa 1976). 2009;34(8): $765-770$

13. Yoshida G, Ushirozako H, Hasegawa T, Yamato Y, Kobayashi S, Yasuda T, et al. Preoperative and postoperative sitting radiographs for adult spinal deformity surgery: upper instrumented vertebra selection using sitting $\mathrm{C} 2$ plumb line distance to prevent proximal junctional kyphosis. Spine (Phila Pa 1976). 2020;45(15):E950-E958.

14. Mao SH, Feng ZX, Qian BP, Qiu Y. The clinical relevance of the presence of bridging syndesmophytes on kyphosis correction and maintenance following pedicle subtraction osteotomy for thoracolumbar kyphotic deformity in ankylosing spondylitis: a comparative cohort study. BMC Musculoskelet Disord. 2018;19(1):97.

15. Cho KJ, Suk SI, Park SR, Kim JH, Kang SB, Kim HS, Oh SJ. Risk factors of sagittal decompensation after long posterior instrumentation and fusion for degenerative lumbar scoliosis. Spine (Phila Pa 1976). 2010;35(17):1595-1601.

16. Debarge R, Demey G, Roussouly P. Sagittal balance analysis after pedicle subtraction osteotomy in ankylosing spondylitis. Eur Spine J. 2011;20(suppl 5):619-625.

17. Kim YJ, Bridwell KH, Lenke LG, Kim J, Cho SK. Proximal junctional kyphosis in adolescent idiopathic scoliosis following segmental posterior spinal instrumentation and fusion: minimum 5-year follow-up. Spine (Phila Pa 1976). 2005; 30(18):2045-2050.
18. Yilgor C, Sogunmez N, Boissiere L, Yavuz Y, Obeid I, Kleinstuick F, et al. Global alignment and proportion (GAP) score: development and validation of a new method of analyzing spinopelvic alignment to predict mechanical complications after adult spinal deformity surgery. J Bone Joint Surg Am. 2017;99(19):1661-1672.

19. MacKay K, Brophy S, Mack C, Doran M, Calin A. The development and validation of a radiographic grading system for the hip in ankylosing spondylitis: the bath ankylosing spondylitis radiology hip index. J Rheumatol. 2000;27(12): 2866-2872.

20. Creemers MC, Franssen MJ, van't Hof MA, Gribnau FW, van de Putte LB, van Riel PL. Assessment of outcome in ankylosing spondylitis: an extended radiographic scoring system. Ann Rheum Dis. 2005;64(1):127-129.

21. Qian BP, Mao SH, Jiang J, Wang B, Qiu Y. Mechanisms, predisposing factors, and prognosis of intraoperative vertebral subluxation during pedicle subtraction osteotomy in surgical correction of thoracolumbar kyphosis secondary to ankylosing spondylitis. Spine (Phila Pa 1976). 2017;42(16):E983-E990.

22. Kim KT, Lee SH, Suk KS, Lee JH, Im YJ. Spinal pseudarthrosis in advanced ankylosing spondylitis with sagittal plane deformity: clinical characteristics and outcome analysis. Spine (Phila Pa 1976). 2007;32(15):1641-1647.

23. Cogniet A, Aunoble S, Rigal J, Demezon H, Sadikki R, Le Huec JC. Clinical and radiological outcomes of lumbar posterior subtraction osteotomies are correlated to pelvic incidence and FBI index: prospective series of 63 cases. Eur Spine J. 2016;25(8):2657-2667.

24. Edwards CC II, Bridwell KH, Patel A, Rinella AS, Jung Kim $\mathrm{Y}$, Berra $\mathrm{AB}$, et al. Thoracolumbar deformity arthrodesis to L5 in adults: the fate of the L5-S1 disc. Spine (Phila Pa 1976). 2003;28(18):2122-2131.

25. Zhao SZ, Qian BP, Qiao M, Jiang J, Qiu Y. Does solid fusion eliminate rod fracture after pedicle subtraction osteotomy in ankylosing spondylitis-related thoracolumbar kyphosis? Spine J. 2019;19(1):79-86.

26. Cho SK, Shin JI, Kim YJ. Proximal junctional kyphosis following adult spinal deformity surgery. Eur Spine J. 2014; 23(12):2726-2736.

27. Weng W, Wu H, Wu M, Zhu Y, Qiu Y, Wang W. The effect of total hip arthroplasty on sagittal spinal-pelvic-leg alignment and low back pain in patients with severe hip osteoarthritis. Eur Spine J. 2016;25(11):3608-3614.

\section{Disclosures}

The authors report no conflict of interest concerning the materials or methods used in this study or the findings specified in this paper.

\section{Author Contributions}

Conception and design: Qiu, Zhao, Qian, Wang. Acquisition of data: Zhao, Qian, Huang, Qiao, Wang. Analysis and interpretation of data: Zhao. Drafting the article: Zhao, Qiao. Critically revising the article: Qiu, Qian, Huang, Qiao, Wang. Reviewed submitted version of manuscript: all authors. Approved the final version of the manuscript on behalf of all authors: Qiu. Statistical analysis: Zhao, Huang. Administrative/technical/material support: Qiu, Zhao. Study supervision: Qiu, Qian.

\section{Correspondence}

Yong Qiu: Affiliated Drum Tower Hospital, Medical School of Nanjing University, Nanjing, China. scoliosis2002@sina.com. 